\title{
Nanoscale Probing of Bandgap States on Oxide Particles Using Electron Energy-Loss Spectroscopy
}

\author{
Qianlang Liu ${ }^{1}$, Katia March² and Peter A. Crozier ${ }^{1}$ \\ 1. School for the Engineering of Matter, Transport and Energy, Arizona State University, 85287, \\ Arizona, USA \\ 2. Laboratoire de Physique des Solides, Bâtiment 510, Université Paris-Sud, 91405 Orsay Cedex, \\ France.
}

In heterogeneous catalysis, nanoparticulate systems are usually preferred due to their large surface areas. The electronic structure on the surfaces/interfaces determines the charge transfer and controls the selectivity and kinetics of the chemical reactions. Heterogeneity and defects very often will occur during the preparation and processing of particulate based catalysts giving rise to different types of extrinsic surface states. Characterizing the local nanoscale variation in oxide surface states is challenging but important for developing a fundamental understanding of functionality. With the recent advances in monochromated scanning transmission electron microscopy (STEM) electron energy-loss spectroscopy (EELS), routine ultra-high energy resolution of $15 \mathrm{meV}$ or better can be achieved with a sub-nanometer electron probe[1]. EELS spectra can be acquired using an aloof beam configuration in which the electron beam is parked several nanometers away from the surface. This dramatically reduces the radiation damage which is important in probing surface electronic structures. In this study, $\mathrm{MgO}$ and $\mathrm{TiO}_{2}$ nanoparticles are selected as model oxides. Extrinsic surface states resulted from the preparation processes are expected to be observed. Interpretation of the EELS spectra using dielectric theory and density of states (DOS) simulations will be discussed.

$\mathrm{MgO}$ was obtained by collecting the product particles while combusting $\mathrm{Mg}$ ribbon in air and then exposing to water vapor. Ni metal was loaded on commercial $\mathrm{TiO}_{2}$ anatase particles (SigmaAldrich) using wetness impregnation followed by high temperature reduction at $450{ }^{\circ} \mathrm{C}$ in flowing $\mathrm{H}_{2}$. An aberration-corrected NION UltraSTEM100 microscope coupled with a monochromator and a Gatan Enfinium spectrometer was employed to acquire all the spectra. The microscope was operated at $60 \mathrm{kV}$ with an energy dispersion of $5 \mathrm{meV}$ per channel. FWHM of the zero-loss peak was better than $25 \mathrm{meV}$. Convergence angle $\alpha$ was $30 \mathrm{mrad}$, and detector collection angle $\beta$ was $15 \mathrm{mrad}$ with $1 \mathrm{~mm}$ EELS entrance aperture.

As shown in Fig.1a, when the electron beam is in bulk $\mathrm{MgO}$, background removed transmission spectrum shows an abrupt intensity increase at $7.3 \mathrm{eV}$ corresponding to the bandgap onset. Interestingly, when beam is $4 \mathrm{~nm}$ away from the $\mathrm{MgO}$ (100) surface, the aloof spectrum shows an additional continuous intensity rise starting from $5.6 \mathrm{eV}$ leading to a plateau which merges into the bandgap onset. The most likely origin of the surface phase is the presence of hydroxyl species leading to a possible thin layer of magnesium hydrate. A dielectric model was employed to simulate the spectrum of a thin $\mathrm{Mg}(\mathrm{OH})_{2}$ surface layer on $\mathrm{MgO}$ [2]. A $2.4 \mathrm{~nm} \mathrm{Mg}(\mathrm{OH})_{2}$ layer gave the best fit to the experiment data shown in Figure 1b. To correlate this feature with specific states in the bandgap, calculations based on DOS data were performed although excitonic effects were not included thus the peak at $7.3 \mathrm{eV}$ is not in the simulation. A broad filled state centered at 1.1 $\mathrm{eV}$ above the valence band with a FWHM of $0.7 \mathrm{eV}$ is found to be responsible for the plateau 
features in the pre-bandgap region (Fig. 1c). In the $\mathrm{TiO}_{2}$ case, a line scan was conducted and only when the beam was close to the surface of the anatase, pronounced peaks were observed in the bandgap (Fig.2a). Using the DOS approach, a sharp half-filled state and an empty state at $1.6 \mathrm{eV}$ and $2.4 \mathrm{eV}$ above the valence band were inserted to simulate the experimental spectrum (Fig.2bc), which is probably arising from two different oxygen vacancy configurations induced in the heat treatment process.

\section{References:}

[1] O.L. Krivanek et al, Nature 514 (2014), 209.

[2] A. Howie; R.H. Milne, Ultramicroscopy. 18 (1985) 427-434.

[3] The support from US Department of Energy (DE-SC0004954) and the use of NION microscope at John M. Cowley Center for HR Microscopy at Arizona State University is gratefully acknowledged.
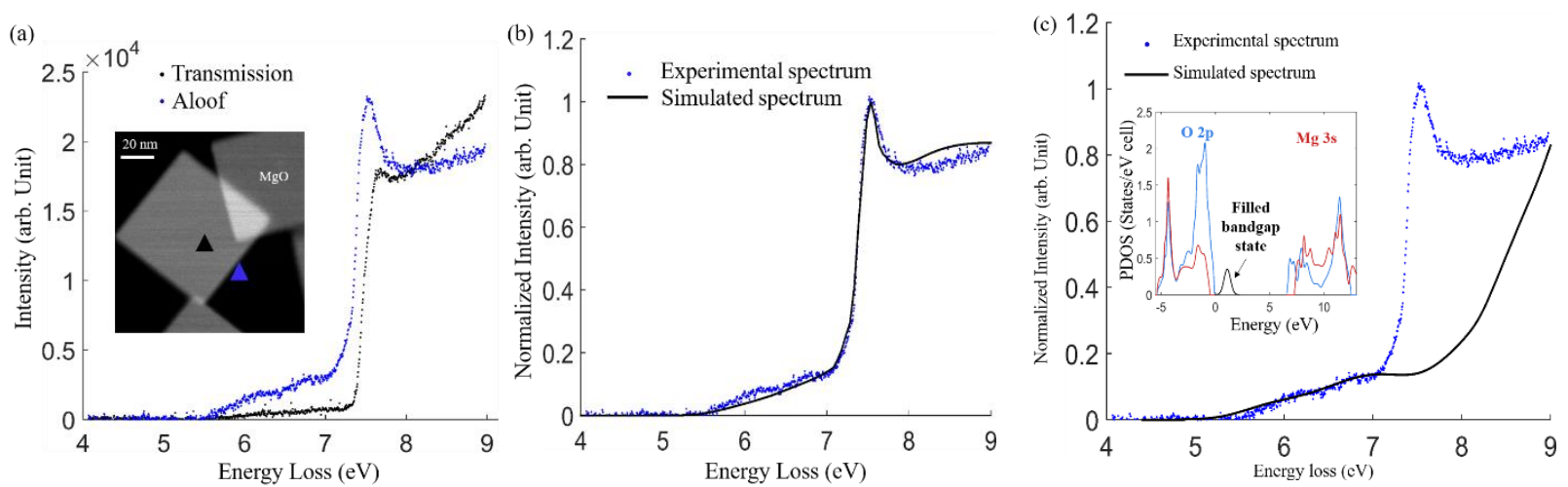

Figure 1: (a) HAADF image shows $\mathrm{MgO}$ nanocubes and beam positions for transmission (black triangle) and aloof (blue triangle) configurations. Background removed transmission and aloof EELS spectra are shown. (b) Simulated aloof EELS spectrum for a $2.4 \mathrm{~nm}$ overlayer of $\mathrm{Mg}(\mathrm{OH})_{2}$ on $\mathrm{MgO}$ compared with the experimental aloof spectrum. (c) Insertion is projected DOS of $\mathrm{MgO}$ with a Gaussian shape filled state $1.1 \mathrm{eV}$ above the valence band with FWHM of $0.7 \mathrm{eV}$.
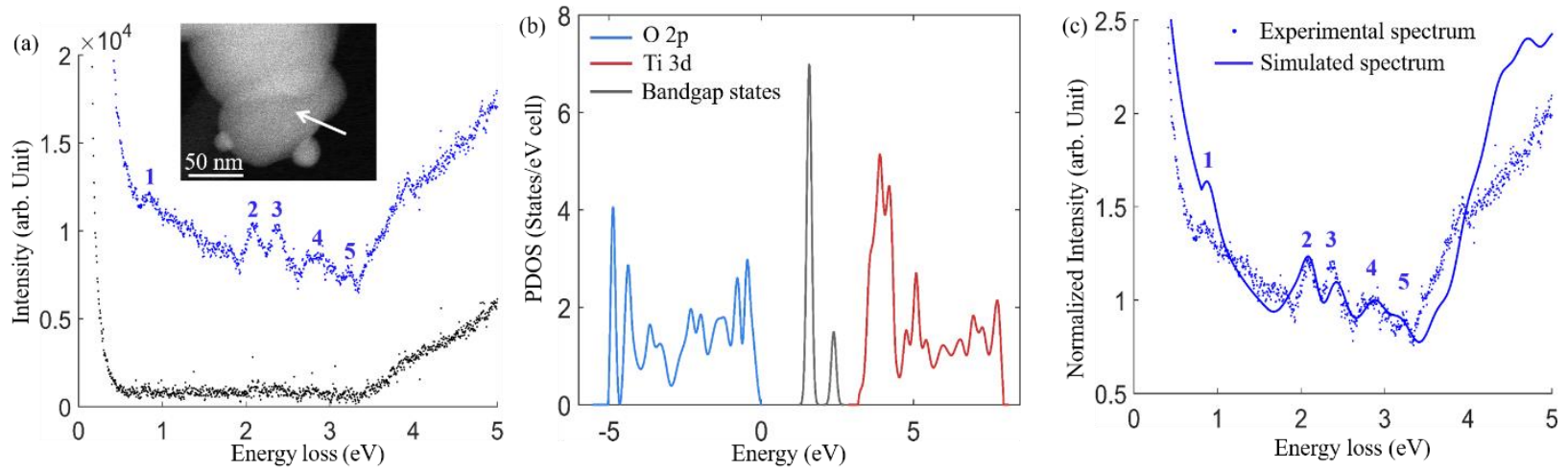

Figure 2: (a) Transmission (black) and aloof (blue) spectra extracted from a line scan going from the vacuum into anatase particle. Insert shows an HAADF image of anatase particle supporting two Ni metal particles. (b) PDOS of anatase with Gaussian shaped states center at 1.575 and 2.380 $\mathrm{eV}$ above the valence band with FWHM of $0.19 \mathrm{eV}$. (c) Simulated spectrum from the DOS in b) overlaid with the experimental spectrum. A simple powerlaw background has been added to the simulation as a guide to the eye for comparing simulation with experiment. 